(1966) estimated that the minimum molecular weight of the $R 17$ RNA synthetase monomer is about 50,000 , which corresponds to roughly 400 amino-acids. If these values are accepted, there is theoretically enough information in the $R 17$ genome to code for a fourth protein of up to about 200 amino-acids since the total $R 17$ genome could code for 1,100 amino-acids. It is very unlikely, however, that a fourth $R 17$ protein, if it exists, is a structural protein.

In the second paper Argetsinger-Steitz reports the large-scale isolation and partial purification of $\mathrm{A}$ protein, which is apparently very insoluble and difficult to work with because it tends to bind to any surface. Nevertheless, she produced 90 per cent pure A protein., and amino-acid analysis of this material indicates that there are five histidine residues per 38,000 molecular weight. The isolation of A protein is a considerable technical achievement, but unfortunately the really interesting questions about the A protein, namely, how it functions and how it confers infectivity on the phage particles, still remain to be answered.

In the same journal (ibid., 947) Argetsinger-Steitz reports the discovery of a curious slowly sedimenting but infective form of $R 17$. This form of the phage is produced by heating normal $R 17$ particles at $46^{\circ} \mathrm{C}$ in a buffer of low ionic strength. The particles produced by this treatment sediment at $45 S-50 S$ but they have not lost either RNA or protein. They are, however, more sensitive to RNase digestion than the normal phage presumably because the RNA is either partially extruded from the particle or else the entire particle is expanded so as to expose the RNA to the external medium. But since the "puffed particles" are infective they cannot have lost the site which is complementary to the phage adsorption site or the host cells, and experiments with ${ }^{3} \mathrm{H}$ histidine labelled phage have shown that A protein is not lost from the slowly sedimenting particles.

\section{Associating Systems}

\section{from our Molecular Biology Correspondent}

IT is by now abundantly clear that a large number of proteins exist in solution in a state of associationdissociation equilibrium. The evaluation of such equilibria by physical measurements in real, non-ideal systems has until quite recently appeared a prohibitively difficult proposition. Some strikingly successful treatments have now, however, been developed, and are beginning to produce important contributions to protein chemistry.

The first comprehensive treatment of indefinite associations has been presented by Adams and Lewis (Biochemistry, 7,$1044 ; 1968$ ). This type of behaviour has been observed with a number of proteins-the most striking recent example being glutamic dehydrogenase; as Eisenberg and Tompkins showed, its molecular weight increases without limit with increasing protein concentration. It has been shown that procedures based on sedimentation velocity experiments are likely to be unreliable because of the hydrostatic pressures generated at high centrifugal fields, which act to annul the volume change commonly accompanying association equilibria. These strictures do not, however, apply to low-speed sedimentation equilibrium, and this is the approach which Adams and Lewis have investigated. Assuming only that the association can be described by a single equilibrium constant. and the non-ideality by a single virial coefficient, they derive an expression for the ratio of the monomer weight to the apparent weight-average molecular weight at any concentration-the latter being the quantity. experimentally obtained at points in the ultracentrifuge cells--in terms of the non-ideality, which can thus be determined. This in turn yields the equilibrium constant by way of one of the basic equations, and further useful relations, involving the moments of the. molecular weight distribution, are given which allow checks for the internal consistency of the parameters. 'The monomer molecular weight must, of eourse. be known.

To show off the precision of their method. Adams and Lewis have applied it to the self-association of $\beta$-lactoglobulin $\mathrm{A}$ at acid $p \mathrm{H}$. The observed equilibrium distribution is shown to be incompatible with a monomer-dimer-octamer system, which had been inferred in earlier work by light-scattering. The fit for an indefinite association, on the other hand, was wellnigh perfect, with a single equilibrim constant of 400 .

A quite different approach is to apply transfer theory to migrating boundaries, eschewing if possible the use of sedimentation velocity: the theory is equally well applied to the experimentally more tractable technique of gel filtration. The theory was extensively developed by Gilbert and co-workers, and an elegant application has now appeared from his laboratory (Chiancone et al. J. Biol. Chem., 243, 1212; 1968). The dissociation equilibrium of oxyhaemoglobin at neutrality and in moderate ionic strength has been studied, and the theoretical principles are explained. The methor consists in applying to a column ('Biogel') a volume of protein solution at fixed concentration and, when this has entered the column, following it with one of higher concentration, so that a stepwise elution profile is obtained. The second solution is followed by solvent, causing elution of a further concentration boundary. The elution volumes of either boundary are related to the concentrations of the constituents, for the proportion of protein entering the column matrix (gel beads) will depend on the relative proportions of the lower and higher molecular weight species present. The results of experiments at different protein concentrations must then be fitted according to possible dissociation schemes and corresponding parameters, and this is a complex process, involving a non-linear least-squares fit. It requires also that the elution volumes for the individual species, as well as their approximate dependence on concentration, be known. 'These values are obtained well enough by the use of myoglobin to represent the monomer, and a well established relation between elution volume and molecular weight. The results are uniquely fitted by a dimer-tetramer equilibrium, the presence of the hypothetical trimer species being formally eliminated. Within the concentration range of the experiments-spanning more than two orders of magnitude-the concentration of the monomer species is too low for useful conclusions. However. for the dimer--tetramer equilibrium, an association constant of some 200 is deduced. Thus, at a protein concentration of $0 \cdot 01$ per cent, half the haemoglobin is dissociater into dimers, whereas at 1 per cent concentration this falls to $6-7$ per cent. 